\section{Conn, Jerome W.}

M. Bidlingmaier

Medizinische Klinik und Poliklinik IV, Klinikum der

Universität München, München, Deutschland

Lebensdaten Geboren 24. September 1907 in New York City; gestorben 11. Juni 1994 in Naples, Florida. Amerikanischer Internist, Endokrinologe. Medizinstudium ab 1925 zunächst an der Rutgers University, seit 1928 an der University of Michigan Medical School in Ann Arbor. Abschluss (MD) 1932. 1935 Assistent Professor, seit 1943 Leiter der Abteilung für Endokrinologie und Stoffwechsel. 1944 Professor für Innere Medizin, seit 1968 LH Newburgh Distinguished University Professor. Conn verbachte seine gesamte akademische Karriere bis zu seiner Emeritierung 1974 an der University of Michigan. Er erhielt für seine Arbeiten eine Vielzahl wissenschaftlicher Auszeichnungen, unter anderem 1958 die Banting-Medaille der American Diabetes Association.

Verdienste Obwohl Conn auch wichtige Studien zur Erforschung des Kohlehydratstoffwechsels beigetragen hat, sind seine wichtigsten wissenschaftlichen Arbeiten die zur Rolle der Steroide in der Regulation von Blutdruck und Salz-Wasser-
Haushalt. 1954 beschrieb er den Fall einer 34 Jahre alten Patientin mit Hypertonie, Hypokaliämie und metabolischer Alkalose. Conn konnte eine erhöhte Konzentration des salzretinierenden Steroids Aldosteron im Urin der Patientin nachweisen und vermutete als Ursache des Mineralokortikoidexzesses einen adrenalen Tumor. In der Tat konnte ein solcher Tumor bei einem operativen Eingriff gefunden und entfernt werden, die Symptome verschwanden. In den folgenden Jahren klärte Conn weitere Teilaspekte des Renin-Angiotensin-Aldosteron-Systems auf und führte unter anderem die bis heute in Form des Aldosteronzu-Renin-Quotienten verwendete Berücksichtigung der Suppression von Renin in die Diagnostik ein. Der inzwischen als häufigste Ursache der sekundären Hypertonie erkannte primäre Hyperaldosteronismus wird nach seinem Erstbeschreiber auch Conn-Syndrom genannt.

\section{Literatur}

Conn JW, Part I (1955) Painting background. Part II. Primary aldosteronism, a new clinical syndrome. J Lab Clin Med 45:3-17

Conn JW, Cohen EL, Rovner DR (1964) Suppression of plasma renin activity in primary aldosteronism. JAMA 190:213-221

National Academy of Sciences (1997) Biographical memoirs, Bd 71. The National Academies Press, Washington, DC. https://doi.org/ $10.17226 / 5737$ 